\title{
Clustering and Cooperative Beamforming in Green Cognitive Radio Networks with Relays
}

\author{
Ashok S Kumar \\ Electronics and Communication Engineering \\ NSS College of Engineering \\ Palakkad, Kerala, India
}

\author{
Sudha T. \\ Electronics and Communication Engineering \\ NSS College of Engineering \\ Palakkad, Kerala, India
}

\begin{abstract}
A relay assisted cooperative beamforming technique can efficiently improve the spectral efficiency and can reduce the interference to primary users in a cognitive underlay systems. Our objective is to reduce the total power cost of Secondary Users (SU) while limiting the interference in the direction of Primary Users (PU). Kmean algorithm which is a partition based clustering algorithm is used here as the clustering algorithm. While considering flexible cooperation as general scheme, we collectively optimize the clustering and the beamforming to lower the overall power consumption while fulfilling the secondary users quality of service and satisfying the primary users interference temperature limits. An iterative algorithm called generalized Benders decomposition method is used to find an optimal solution. The indirect transmission mode of relays is considered in this work. The relays are equipped with directional antennas which will reduce the interference to the PU and meet the power cost requirement of SU under the assumptions that the clusters are non-overlapping and fixed. Thus spectral efficiency can be increased. Through simulations, we analyzed the advantages of algorithms and showed the benefits of the relays in cognitive wireless networks.
\end{abstract}

\section{General Terms}

Cognitive Radio, Energy Efficiency

\section{Keywords}

Green Networks, Cognitive Radio, Cooperative Beamforming, Kmean algorithm, Benders decomposition algorithm, Indirect relay mode

\section{INTRODUCTION}

$\mathrm{RF}$ spectrum is a limited natural resource and its proficient use is of very important. Over the past two decades there is an enormous progression in wireless communication, and we are now living in a world where there are ever-increasing numbers of wireless communication devices in operation. Cognitive Radio which was first proposed in late 1990's has been renowned as a suitable way to ameliorate spectrum efficiency of wireless communications by exploiting under-utilized licensed spectrum in frequency,spatial and temporal domains. Cognitive radio(CR) is a wireless communication system which is attentive of the environment and its changes and can adapt its transmission/receiver parameters accordingly with the objective of reliable communication whenever and wherever needed [1]-[4]. Green communication is energy efficient communication. Cognitive Radio increase spectral efficiency by giving opportunistic access of the frequency bands to other users, thus less power can be used for a certain transmission rate.

An overview of cooperative spectrum sensing has been studied in [5]. Cooperative sensing declines the probabilities of miss-detection and false alarm significantly. It can solve hidden primary user problem and it can decrease sensing time. Due to large-scale fading (radio shadows), it is very difficult to reach adequate sensitivity in a single mobile station or access point [6]. Further, in cooperative sensing, different stations are in different radio environments, and some of them might be in particularly good position for securing each specific spectrum hole. In our work we initialized many nodes (secondary users) and some of the nodes can access spectrum hole easily. Due to the above mentioned advantages, we applied cooperative spectrum sensing as the general method to detect spectrum hole. In our endeavor we use centralized cooperative spectrum sensing method. In this, there is a Cluster Head $(\mathrm{CH})$ node that collects data from the other nodes. We divide the nodes into different clusters and selects a $\mathrm{CH}$ for each clusters according to clustering algorithm

The performance of Cooperative Beamforming has been investigated extensively in [7]. This technique can upsurge the range of the communication link, since the signal beam is concentrated only to the direction of the communication partner so that no energy is squandered to the other direction. So in order to reduce the interference to PU, we have adopted this technique in our work.

The work reported in [8] exploited the advantages of using Kmeans as the clustering algorithm. K-means algorithm reduces the complexity of clustering and it is a simple technique for estimating the mean of a set of $\mathrm{K}$ groups. In K-means algorithm number of cluster $\mathrm{K}$ must be defined beforehand and it can be applied only when the mean of a cluster is defined. K-means algorithm is used as the clustering algorithm in our work.

Multi-cell cooperation schemes can be classified into three types like Full cooperation, Inter-cell cooperation, Flexible cooperation [9]. While considering flexible cooperation scheme as general case and full cooperation and inter-cell coordination as special cases, we 
collectively optimize the clustering and the beamforming to lower the overall power consumption while fulfilling the secondary users quality of service and satisfying the primary users interference temperature limits. We express this problem as a mixed-integer nonlinear program and decompose it into a master problem and a beamforming sub problem. An iterative algorithm that has been derived based on Benders decomposition in [9],[10] is a way to split complicated mathematical programming problems into two, and thereby simplifying the solution by solving one master problem and one sub problem is used to find the optimal solution in this work.

Amplify-and-forward (AF) relaying used in [11] can progress the sensing performance and increase the throughput. Indirect mode takes place when the direct links between the transmitter and cognitive receivers botched due to some problems such as shadowing, multipath fading and path loss and thus QoS requirements are not fulfilled in the secondary network. Under such conditions, relaying process initiates and directional relays are responsible to direct the message signal from the secondary transmitter to the SU. The relay technique used in our work can tackle the problem of interference to the PU and meet the power cost requirement of SU and tries to assure the desired QoS in both primary and secondary networks. The directional relays and omni directional receiving capability can significantly improve system performance [12],[13].

In this work, we make the assumptions like perfect synchronization and perfect channel information for the cooperative beamforming. The location of each node is chosen randomly following a uniform distribution. Each node is equipped with a single ideal isotropic antenna. There are no reflections or scattering, thus there is no multipath fading or shadowing. The nodes are sufficiently separated so no antenna coupling occurs. There is no mutual coupling between different antennas and cognitive radio user nodes. The nodes location is static, meaning that the location does not change over time.

This paper is organized as follows: Section II exposes a detailed study of the Network analysis of the proposed system, and Section III discusses the simulation results for its total power cost of SU's and the total interference to the PU. Finally, the conclusions are briefed in Section IV.

\section{NETWORK ANALYSIS}

Full cooperation is used when all Base Stations (BS) share their antennas to cooperatively serve their users. Total power cost of full cooperation is high. Inter-cell coordination is the simplest strategy in which each user is served by a single BS. Flexible cooperation is the scheme in which each user is served either by a single BS or by multiple cooperating ones.

Here we consider cognitive underlay system in which the secondary users are represented by $S=s_{1}, s_{2}, \ldots, s_{|S|}$ and primary users as $V=v_{1}, v_{2}, \ldots, v_{|V|}$. Cognitive BSs, $B=b_{1}, b_{2}, \ldots, b_{|B|}$ has $m$ antennas each. In order to model the system let us define a binary vector:

$$
Z=\left(z_{b s}\right)_{b \epsilon B, s \epsilon S} \epsilon\{0,1\}^{|B| \cdot|S| \times 1}
$$

The component $z_{b s}$ of $Z$ determines whether BS, $b$ serves the secondary user, $s$ or not.

$$
z_{b s}= \begin{cases}1 & \text { if user } \mathrm{s} \text { is served by } B S \\ 0 & \text { otherwise }\end{cases}
$$

In full cooperation each user can be served by many BSs. The special case of full cooperation is:

$$
z=1_{|B| \cdot|S| \times 1}
$$

in which each user is collectively served by all BSs. The special case of inter-cell coordination corresponds to:

$$
\sum_{b \in B} z_{b s}=1, \quad \forall s \epsilon S
$$

which ensures that each user is assigned to only one BS.

Assuming full cooperation. Let $h_{s} \triangleq\left(h_{b s}\right)_{b \in B} \in C^{m|B| \times 1}$ represent the channel vector from the distributed antennas of all BSs to the receiving antennas of a secondary user, $s . h_{b s} \epsilon C^{m \times 1}$ is the channel between BS, $b$ and user, $s$. The channel vector $h_{b s}$ corresponds to both Rayleigh fading and path loss attenuation. Similarly, $v_{s} \triangleq\left(v_{b s}\right)_{b \in B} \epsilon C^{m|B| \times 1}$ represents the array beamforming vectors associated with user, $s$ and $v_{b s} \epsilon C^{m \times 1}$ is the beamforming vector of BS, $b$ for user, $s$. If we consider the special case of full cooperation in which each user, $s$ is served by all BSs, its received signal is represented as:

$$
y_{s}=h_{s}{ }^{H} v_{s} d_{s}+\sum_{j \in S \backslash\{s\}} h_{s}^{H} v_{j} d_{j}+n_{s}
$$

where $(.)^{H}$ represents the conjugate transpose, $n_{s}$ is a complex zero mean and $d_{s}$ represents the data symbol of user, $s$ that is transmitted by BSs through the channel $h_{s}$. The signal-to-interferenceplus-noise ratio of user, $s$ is given by:

$$
S I N R_{s}=\frac{\left|h_{s}^{H} v_{s}\right|^{2}}{\sum_{j \in S \backslash\{s\}}\left|h_{s}^{H} v_{s}\right|^{2}+\sigma_{s}^{2}}
$$

$\sigma_{s}^{2}$ is the variance. Achievable rate is given by the equation:

$$
R_{s}=W\left(1+\frac{S I N R_{s}}{\Gamma}\right)
$$

where $W$ and $\Gamma$ represents the channel bandwidth and the SINR gap between the rate achieved in practical case and Shannon capacity. The transmit power, $P_{b}=\sum_{s \in S}\left\|v_{b s}\right\|_{2}^{2}$ of each BS, $b$ should not exceed a practical power limit:

$$
\sum_{s \in S}\left\|v_{b s}\right\|_{2}^{2} \leq P_{b}^{\max }, \quad \forall b \in B
$$

For flexible cooperation, we also add a constraints:

$$
\left\|v_{b s}\right\|_{2}^{2} \leq z_{b s} P_{b}^{\max }, \quad \forall(s, b) \epsilon S \times B
$$

The role of the additional constraints is to make sure that when a specific BS, $b$ does not serve a specific user, $s$, the beamforming vector $v_{b s}$ should be a zero vector. SINR definition initially assumes full cooperation, after we add the constraints it is also valid for flexible cooperation. In the cooperative cognitive underlay system the secondary BSs shares the same channel with the primary 
system under the condition that it should not cross the interference temperature limit $I_{v} \geqslant 0$ imposed by each primary user as [9]:

$$
\sum_{b \in B} \sum_{s \epsilon S}\left|g_{b v}^{H} v_{b s}\right|^{2} \leq I_{v}, \quad \forall v \epsilon V
$$

$g_{b v}$ is the channel from secondary BS, $b$ to the primary user, $v$ :

$$
p_{t o t}=p_{t x}+p_{s p}+p_{b}+p_{o}
$$

where $p_{t o t}, p_{t x}, p_{s p}, p_{o}, p_{b}$ denote the total power consumption, the transmit power radiated by all BSs, the signal processing power cost,the fixed power cost and the backhaul power cost respectively. The fixed power $p_{o}$ in is the sum of fixed power consumed by all BSs:

$$
p_{o}=\sum_{b \in B} y_{b} p_{\text {stat }}
$$

$y_{b}$ is the binary variable which defines the activity status of BS $b$ such that:

$$
y_{b}=\left\{\begin{array}{l}
1 \quad \text { if BS bis serving atleast one user } \\
0 \quad \text { otherwise }
\end{array}\right.
$$

Inorder to collectively optimize clustering and beamforming vectors that minimize the overall power consumption of the secondary system and interference power constraints at the primary users, an iterative algorithm based on the generalized Benders decomposition method in [9],[10], is used to find an optimal solution.

\subsection{Problem Formulation}

The SU's performance in any CR system depends on the factors such as PU's activity and availability of spectrum etc. Here we consider SU as nodes. One of the SU is selected as the $\mathrm{CH}$ according to the selection procedure. The communication between two nodes via $\mathrm{CH}$ is considered here. When $\mathrm{SU}$ is transmitting and during this time if PU also starts transmition, then SU has to decrease its power, otherwise it will cause interference to PU. In this work, our objective is to reduce the total power cost of SU while limiting the interference in the direction of PU.

\subsection{Indirect Mode Relay Equipped with Directional Antennas}

The problems encountered in section 2.1 can be tackled by the introduction of relay node in this model. The network applies a scheme consists of two transmission modes: direct mode and indirect (relay) mode [11]. In direct mode, joint power allocation and beamforming scheme is performed in the secondary transmitter. In this mode, the direct links should assure QoS requirements of the primary network while trying to maximize total SINR in the SUs. In the later mode, which is triggered if QoS requirements are not maintained in at least one SU, directional relays cooperate in transmission process and try to satisfy QoS requirements in both primary and secondary networks. In other words, indirect mode takes place when the direct link from cognitive transmitter to receiver fails and the process of relaying starts. The SINR value of the relay is compared with a predefined threshold and those relays which will have the value of SINR greater than or equal to the threshold value is chosen to participate at the indirect mode [11]. The received signal of the relay node can be expressed as:

$$
y_{r}=\sum_{l=1}^{L} \sqrt{T_{p l}} w_{l}^{H} c_{r} e_{l}\left(t-\tau_{l}\right)+\sqrt{g_{r}^{p} T_{p 0}} e_{0}\left(t-\tau_{0}^{r}\right)+n_{r}(t)
$$

$r=1, \ldots, N, N$ is the number of relay node. $l=1, \ldots L, L$ is the number of receivers. $\tau_{l}$ is the time delay in arriving the message signal to $r_{t h}$ relay from secondary transmitter. $\tau_{0}^{r}$ indicates the time delay of the message signal received from primary transmitter to $r_{t h}$ relay node. $e_{0}(t)$ and $e_{l}(t)$ are the message signal transmitted by the primary transmitter and message signal transmitted by the Secondary transmitter received at $l_{t h}$ Secondary user. Similarly $T_{p l}, T_{p 0}$ represents the transmission power of the secondary transmitter to the $l$ th SU, continuos power transmitted by primary transmitter respectively. $w_{l}$ represent the optimization parameter, $c_{r}$ is the channel response from cognitive transmitter to the $r_{t h}$ relay. $n_{r}(t)$ is the noise at the $r_{t h}$ relay node. $g_{r}$ is the channel loss from primary transmitter to the relay node.

SINR value of the relay for the indirect mode is given by:

$$
\begin{gathered}
S I N R_{r}{ }^{l}=\frac{\left|w_{l}^{H} c_{l}\right|^{2} T_{p l}}{\sum_{i=1, i \neq l}^{L}\left|w_{l}^{H} c_{i}\right|^{2} T_{p i}+\sigma^{2}+k_{r}^{p} T_{p 0}} \\
l=1, \ldots, L
\end{gathered}
$$

$L$ is the number of receivers, $i \epsilon[1, L]$

where $S I N R_{r}{ }^{l}$ is the SINR value of the relay node of the $l_{t h}$ cluster, $\sigma^{2}$ is the noise variance, $k_{r}^{p}$ is the channel loss from primary transmitter to relay node. $T_{p i}$ is the transmission power of the secondary transmitter to the $i_{t h}$ SU. $c_{l}$ is the channel response from cognitive transmitter to the $l_{t h} \mathrm{SU}$ and $c_{i}$ is the channel response from cognitive transmitter to the $i_{t h}$ SU. After determining those relays that are involved in indirect transmission,the relaying phase starts to meet the QoS requirement of SUs. In the indirect mode,the received signal at the $l_{t h} \mathrm{SU}$ can be expressed as [11]:

$$
\begin{aligned}
y_{l}=\sum_{r, \forall r \epsilon R} & \sqrt{k_{l}^{r} T_{p r}} F_{r}\left(\Theta_{1}\right) e_{r}\left(t-\tau_{r}^{l}\right) \\
& +\sqrt{k_{l}^{p} T_{p 0}} e_{0}\left(t-\tau_{0}^{l}\right)+n_{1}^{t}, \quad \forall l \epsilon L
\end{aligned}
$$

Also $k_{l}^{r}$ and $k_{l}^{p}$ are the channel losses between relay and the $l_{t h} \mathrm{SU}$ and between the Primary transmitter and the $l_{t h}$ SU respectively. The parameters $T_{p r}$ and $e_{r} t$ indicates transmission power and message signal of the relay in the relaying phase. $\tau_{r}^{l}$ is the time delay of the message signal received at the $l_{t h} \mathrm{SU}$ and $\tau_{0}^{l}$ indicates the time delay of the message signal received from primary transmitter to $l_{t h}$ SU. $n_{1}(t)$ is the noise at $l_{t h} \mathrm{SU}$. The radiation pattern of the relay can be expressed as:

$$
F_{r}(\Theta)= \begin{cases}1 & \Theta_{r}^{1} \leqslant \Theta \leqslant \Theta_{r}^{2} \quad ; r=1, \ldots, N \\ 0 & \text { otherwise }\end{cases}
$$

where $F_{r}(\Theta)$ is the radiation pattern of the relay in the direction of $l$ th SU.

\subsection{K-Means Clustering algorithm to find $\mathrm{CH}$}

$\mathrm{K}$-means is a partition based clustering algorithm in which it splits the data objects into $\mathrm{k}$ partitions or groups. The grouping is done by minimizing the sum of squares of distances between data and the corresponding cluster centroid. K-means uses the squared Euclidean distance to allocate objects to clusters. K-means returns distances from each point to every centroid. The algorithm description is given below.

Steps for K-Means Clustering algorithm

1) Choose initial cluster seeds at random. These represent the temporary means of the clusters. 


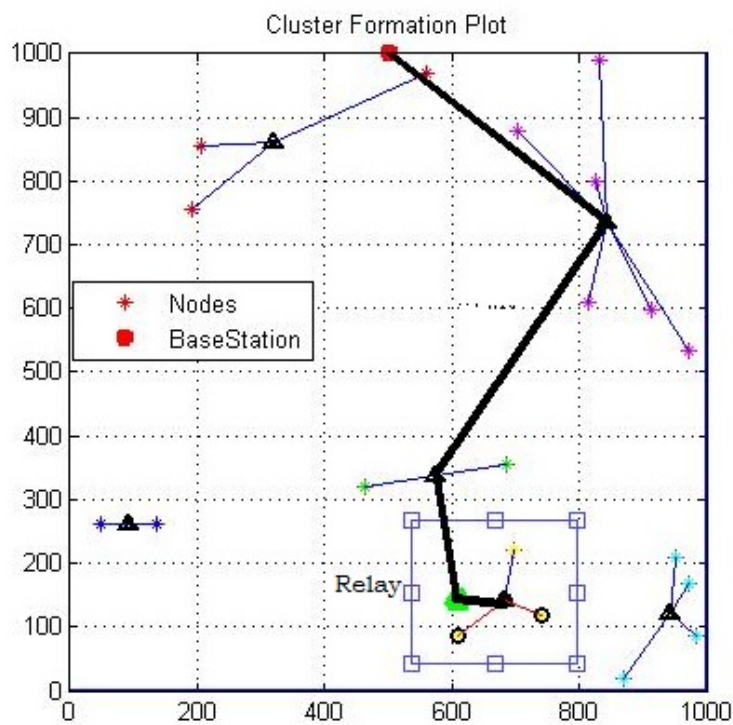

Fig. 1. Cluster formation plot with one relay

2) Compute the squared Euclidean distance from each object to each cluster and each object is assign to the closest cluster

3 ) Find out the new centroid for each cluster and each seed value is replace by the respective cluster centroid

4) Compute the squared Euclidean distance from an object to each cluster and the object is assign to the cluster with the smallest squared Euclidean distance.

5) Recalculate the cluster centroids based on the new membership assignment.

6) Repeat the steps 4 and 5 until no object moves clusters.

\section{SIMULATION RESULTS}

For the numerical simulation, we consider 20 nodes and $6 \mathrm{CH}$ under the assumption that the users are uniformly distributed and non-overlapping. The $\mathrm{CH}$ holds the information like location of the nodes, number of nodes and energy of those nodes that are involved in the specific cluster. For simplicity, we makes the assumption that SINR target are same for all users, i.e. $\rho_{s}=\rho, \forall_{s}$.

Fig. 1 and Fig. 2 depict the cluster formation plot with one relay and two relays respectively. The $\mathrm{CH}$, which holds the information about the specific group is indicated by smaller triangle (black) and Relay Node by bigger triangle (green). BS is denoted by circle, which is located at the upper boundary of the square. The total interference temperature limit is taken as $I_{v}=3 \mathrm{~dB}$ above the noise level. In our work we consider two cases. For the first case, the first relay node (Fig. 1) is selected in between the first two $\mathrm{CH}$ that are involved in the process of transmission and for the second case one of the $\mathrm{CH}$ that is involved in the transmission process as the Relay Node (Fig. 2). Whenever a specific node has to transmit, $\mathrm{CH}$ which has the information regarding the intended node will choose the easiest path to reach the BS. The relay node which has directional transmission capability collects the information from first $\mathrm{CH}$ since it has omni directional receiving capability and forwards it to the second $\mathrm{CH}$ (Fig. 1). For the second case, the first

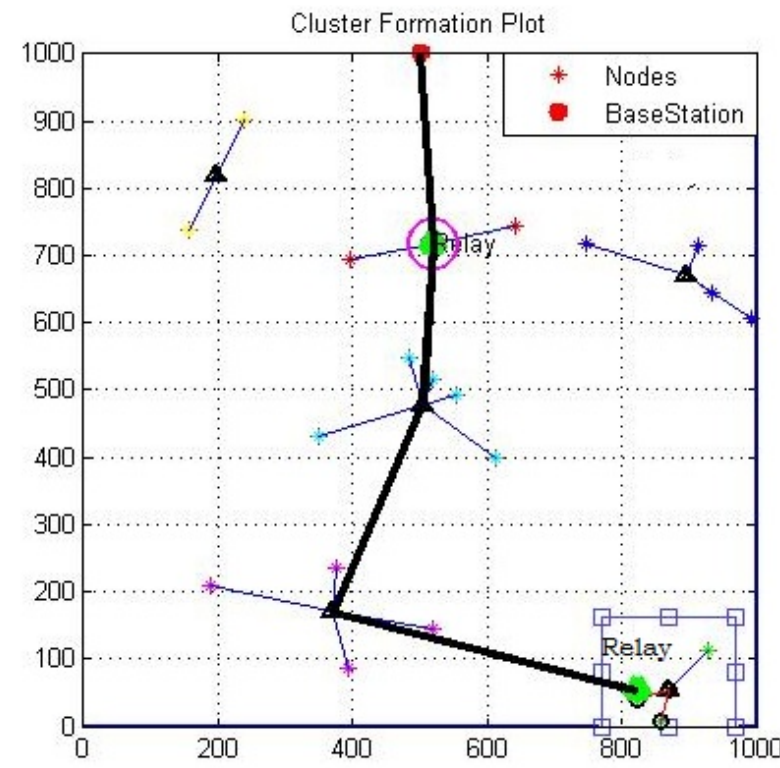

Fig. 2. Cluster formation plot with two relays

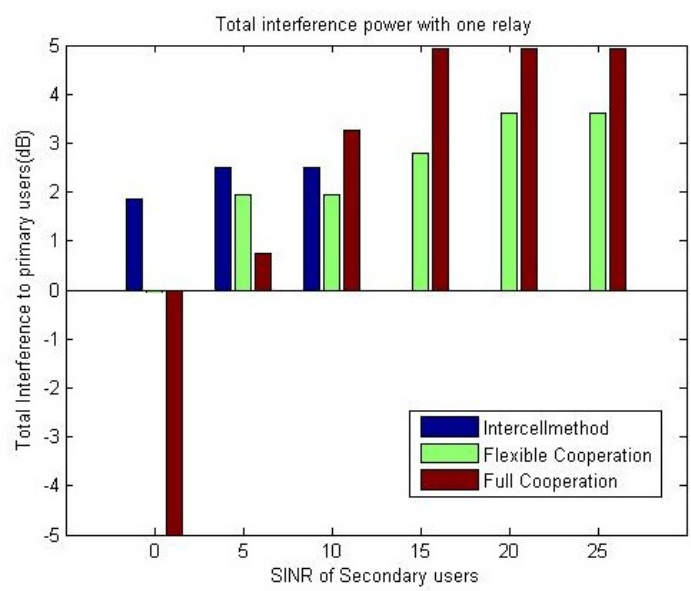

Fig. 3. Total interference to primary users with one relay

relay node forwards the signal to the second $\mathrm{CH}$, then to the next $\mathrm{CH}$ and so on to reach the BS. Here one of the $\mathrm{CH}$ acts as the relay node. The other clusters which are not involved in the process of transmission are considered as primary users.

Fig. 3 and Fig. 4 depict the total interference to primary users with one and two relays. We compared the performance of flexible cooperation, inter-cell cooperation and full cooperation schemes. Interference to primary user increases with SINR value. In the case of Full cooperation scheme, the interference to primary user is less for values of $\rho<10 d B$ and increases afterwards as compared to other two multi-cell cooperation schemes. The simple inter-cell cooperation is optimal at low SINR value. It is no longer feasible when $\rho>10 \mathrm{~dB}$. With the help of relay, the probability of signal to reach the destination increases. Since the relay has directional transmission capability, it will direct the signal to the intended 


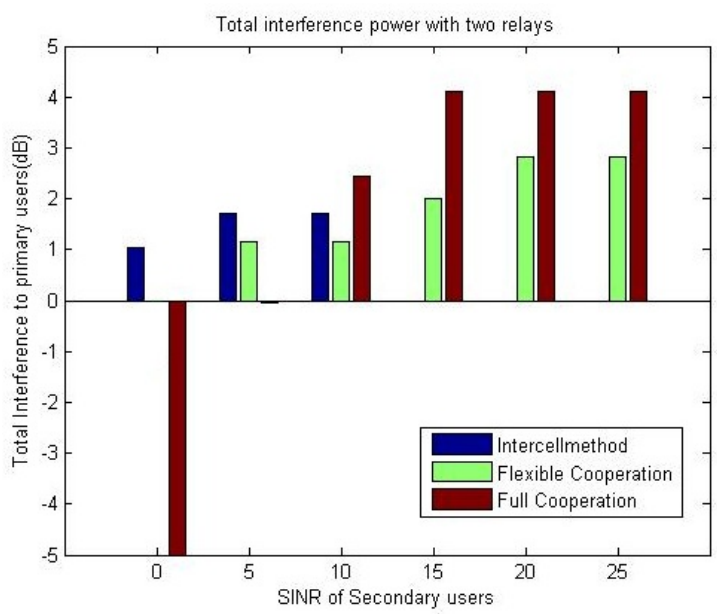

Fig. 4. Total interference to primary users with two relays

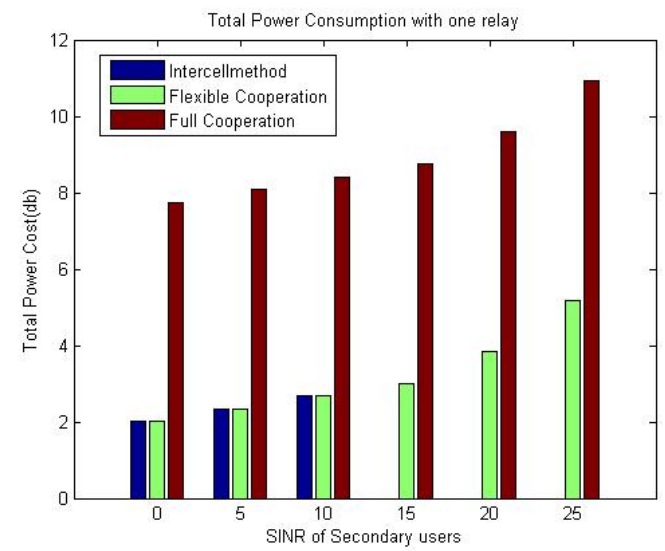

Fig. 5. Total Power cost with one relay

secondary user thereby reduces the interference to primary user. So there will be an overall reduction for the total interference to primary users with relays for the above mentioned cooperation schemes [9].

Fig. 5 and Fig. 6 show the total power cost with one and two relays respectively. Full cooperation has the highest power cost in terms of overhead for sharing user data and channel information through the backhaul network. This scheme achieves the lowest transmit power. The total power cost is the sum of backhaul power, overhead power, transmit power, signal processing power and fixed power cost of all clusters involved in transmission process [9]. While incorporating relay, which has less overall power cost, the total power cost for the three schemes can be reduced. Since the $\mathrm{CH}$ acts as the relay node, the fixed power cost can be reduced and thereby total power cost can be reduced.

\section{CONCLUSION}

Cooperative spectrum sensing improves sensing performance in cognitive radio significantly. In cooperative spectrum sensing concurrent transmission of both primary and secondary can be

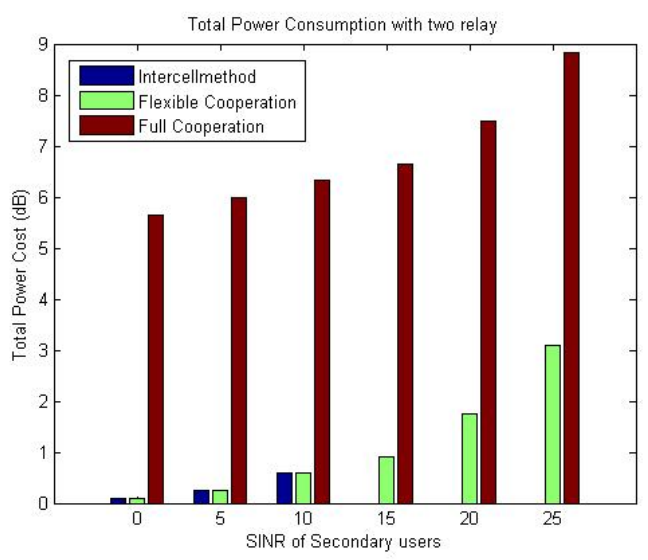

Fig. 6. Total Power cost with two relays

done without causing interference to primary users. The clustering algorithm used is this paper is K-means, which is a partition based algorithm. We consider flexible cooperation as general scheme, we collectively optimize the clustering and the beamforming to lower the overall power consumption while fulfilling the secondary users quality of service and satisfying the primary users interference temperature limits. An iterative algorithm called generalized Benders decomposition method is used to find an optimal solution. We assert that cooperative relay technology is considered as an effective method to improve the performance of both spectrum sensing and secondary transmission. We have investigated the performance of the system using relay technique. Our simulation results showed the benefit of using relay in cooperative cognitive cellular networks for reducing total power cost and interference to primary users.

There are several drawbacks of K-means algorithm like number of clusters and mean has to be defined beforehand. In our future work, we will focus on more powerful optimization algorithm which has fast convergence, simplicity, less control parameters and robustness.

\section{Acknowledgment}

This work is supported by AICTE, Govt. of India through the project grant under Research promotion Scheme No.20/AICTE/RIFD/RPS (POLICY-1)59/2013-14.

\section{REFERENCES}

[1] Ian F, Akyildiz, Won-Yeol Lee,Mehmet C. Vuran, Shantidev Mohanty, NeXt Generation/Dynamic Spectrum Access/Cognitive Radio Wireless Networks: A Survey, Computer Networks,vol. 50, no. 13,(2006):2127-2159.

[2] K. C. Chen, Y. J. Peng, N. Prasad, Y. C. Liang, and S. Sun, Cognitive Radio Network Architecture: Part I - General Structure, ACM International Conference on Ubiquitous Information Management and Communication (ICUIMC) (2008).

[3] Simon,Haykin, Cognitive Radio: Brain-Empowered Wireless Communications,Selected Areas In Communications, IEEE journal on 23.2(2005):201-220

[4] Shrikrishan Yadav, Santosh Kumar Singh, Krishna Chandra Roy, A Smart and Secure Wireless Communication System: 
Cognitive Radio, International Journal of Soft Computing and Engineering (IJSCE) ISSN:Vol.2, Issue-1,(2012):2231-2307

[5] Ian F. Akyildiz, Brandon F. Lo, Ravikumar Balakrishnan, Cooperative spectrum sensing in cognitive radio networks: A survey, Physical Communication 4 (2011):40-62.

[6] Xiaoming Chen, Hsiao-Hwa Chen, and Weixiao Meng, Cooperative Communications for Cognitive Radio Networks:From Theory to Applications, IEEE Communications surveys and tutorials, IEEE Communications surveys and tutorials, vol.16, no.3, (2014):1180-1192.

[7] Yu Zhang,Anese and Georgios B, Distributed Optimal Beamformers for Cognitive Radios Robust to Channel Uncertainties, IEEE transactions on signal processing, IEEE transactions on signal processing, vol.60, issue 12,(2012):6495-6508.

[8] Tapas Kanungo, David M. Mount, Nathan S. Netanyahu, Christine D. Piatko, Ruth Silverman, and Angela Y. Wu, An Efficient k-Means Clustering Algorithm: Analysis and Implementation, IEEE transaction on pattern analysis and machine intelligence, vol.24, no.7,(2002):881-892.

[9] Rindranirina, Ramamonjison, Alireza Haghnegahdar and Vijay K. Bhargava, Joint Optimization of Clustering and Cooperative Beamforming in Green Cognitive Wireless Networks, IEEE Transaction on wireless communication, vol.13,no.2,(2014) :982-997.

[10] J. Benders, Partitioning procedures for solving mixedvariables programming problems, Computational Management Science, vol. 2, no. 1, (2005):3-19, Available: http://dx.doi.org/10.1007/s10287-004-0020-y

[11] Narges Naouri, Narges Noori, Directional Relays for MultiHop Cooperative Cognitive Radio Networks, Radioengineering, vol.22, no.3,(2013):791-799

[12] Yulong Zou, Jia Zhu, Baoyu Zheng, and Yu-Dong Yao, An Adaptive Cooperation Diversity Scheme With Best-Relay Selection in Cognitive Radio Networks, IEEE transactions on signal processing,vol. 58, no. 10,(2010):5438-5445.

[13] Ali Afana, Vahid Asghari, Ali Ghrayeb, and Sofi'ene Affes, On the Performance of Cooperative Relaying SpectrumSharing Systems with Collaborative Distributed Beamforming, IEEE transactions on communications, IEEE transactions on communications, vol. 62 , no. 3, (2014):857-871. 